\title{
Axonal release sites discovered
}

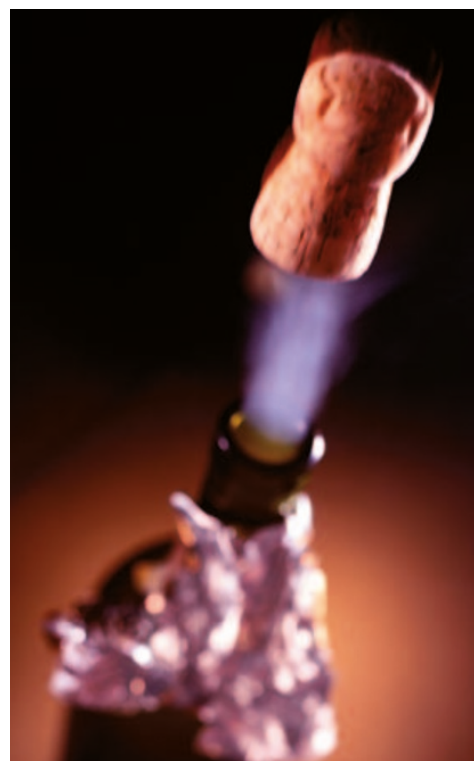

Vesicular neurotransmitter release is the hallmark of fast excitatory synaptic transmission. It was assumed that the machinery required to control this process is restricted to presynaptic membranes in the grey matter. However, recent data have indicated that glutamatergic signalling may also occur in the white matter. Two groups now report the existence of vesicular glutamate release at axonal membranes in the corpus callosum.

The authors of both reports carried out patch-clamping studies on oligodendrocyte precursor cells (OPCs) in slices of the rat corpus callosum. They recorded transient inward currents in response to electrical stimulation of callosal axons, which indicates that action potentials induce axonal glutamate release. Characterization of the currents showed that they were mediated by AMPARs ( $\alpha$-amino-3-hydroxy-5- methyl-4-isoxazole propionic acid receptors) on OPC membranes. The results of both groups suggested that, in the developing and juvenile rat, only calcium-impermeable AMPARs were involved in generating these currents; however, Ziskin et al. showed that, in the mature brain, calcium-permeable AMPARs were also activated. Both studies indicated that unmyelinated axons were principally responsible for generating these currents.

Kukley et al. demonstrated that neurotransmitter release was sustained during repetitive stimulation, indicating that specialized axonal sites are set up for this purpose. Using electron microscopy, both groups observed accumulation of vesicles close to the axonal membrane at sites of contact with OPCs. In addition, Ziskin et al. used immunogold labelling to show that these contacts were glutamatergic and structurally similar to grey matter synapses.

The rapid kinetics of the currents generated in the OPCs indicate that glutamate is released by vesicle fusion. Ziskin et al. showed that pharmacologically enhancing vesicle fusion, or inhibiting vesicle filling, increased and decreased, respectively, the amplitude of the currents in OPCs. Furthermore, Kukley et al. demonstrated that endo- and exocytotic vesicle recycling takes place, by bathing the axons in a fluorescent dye that was taken up into and then released from vesicles.

The axonal neurotransmitter release shares several features with traditional synaptic neurotransmission. Kukley et al. showed that axonal voltage-gated calcium channels can elevate intracellular calcium concentrations sufficiently to trigger vesicle release, and Ziskin et al. showed that blocking calcium channels abolished release. The relationship between calcium levels and transmitter release was found to be similar to that observed at synapses. Transmitter release was highly synchronized after action potential generation, indicating that the vesicles are in close proximity to the calcium microdomain that surrounds these channels. Ziskin et al. also demonstrated that glutamate release from the axons was facilitated by repeated stimulation, and depressed by activation of axonal autoreceptors - characteristic features of synaptic neurotransmission.

Kukley et al. also observed vesicular neurotransmitter release in the optic nerve, suggesting that this process may be widespread in white matter. These studies indicate that vesicular neurotransmitter release is not restricted to grey matter synapses. The functional consequences of vesicular neurotransmitter release in the white matter are unknown; however, concentration gradients of glutamate might be used by the OPCs to guide OPC development, or to control myelination in an activitydependent manner. Furthermore, glutamate release might contribute to excitotoxicity in the white matter following ischaemia.

Katherine Whalley

ORIGINAL RESEARCH PAPER Kukley, M., Capetillo-Zarat, E. \& Dietrich, D. Vesicular glutamate release from axons in white matter. Nature Neurosci. 11 Feb 2007 (doi:10.1038/nn1850) | Ziskin, J. L., Nishiyama, A., Rubio, M., Fukaya, M. \& Bergles, D. E. Vesicular release of glutamate from unmyelinated axons in white matter. Nature Neurosci. 11 Feb 2007 (doi:10.1038/nn1854) 